\title{
14 years of Spitzer publications: data use and reuse
}

\section{Elena Scire}

Elena Scire, "14 years of Spitzer publications: data use and reuse," Proc. SPIE 10704, Observatory Operations: Strategies, Processes, and Systems VII, 1070417 (10 July 2018); doi: 10.1117/12.2312912

SPIE Event: SPIE Astronomical Telescopes + Instrumentation, 2018, Austin, Texas, United States 


\title{
14 Years of Spitzer Publications: Data Use and Reuse Elena Scire ${ }^{\mathrm{a}^{*}}$ \\ ${ }^{a}$ Spitzer Science Center, Caltech M/S 314-6 1200 E. California Blvd, Pasadena, CA 91125;
}

\begin{abstract}
After 14 years of operations, the Spitzer Space Telescope has over 7800 refereed publications, which are catalogued in the Spitzer Bibliographical Database. After depleting the cryogen onboard in 2009 observatory operations were altered in order to run the mission on a budget totaling less than $1 / 3$ that of the cryogenic mission. These changes included decreasing the number of approved science programs and funding to the community while encouraging very large programs (>1000 hours). We present here insights on how Spitzer's operational changes have altered how people publish the data, and present metrics about data use and re-usage for the entire mission in published papers. Spitzer is a community driven observatory and our observing programs vary in number of hours and number of observations depending on the science, and so a comparison between various methods of presenting publication statistics is discussed.
\end{abstract}

Keywords: Spitzer, publications, archives

\section{INTRODUCTION}

\subsection{The Spitzer Space Telescope}

The Spitzer Space Telescope ${ }^{1}$ launched in August of 2003 with three instruments: IRAC (3.6, 4.5, 5.6 and 8.0 micron imaging), MIPS (24, 70, 160 micron imaging) and IRS (5.2 to 38 micron spectra, 16 and 22 micron imaging). Its cryogenic mission was completed in May 2009 with the depletion of the liquid helium coolant on board. Since that time the Spitzer Warm and Beyond Missions (hereafter refereed to at the Warm Mission) has been ongoing with the two shortest wavelength channels of the IRAC camera (3.6 and 4.5 microns). The Spitzer Warm Mission budget is $<1 / 3$ of the cryogenic budget, and operational changes were made to run the telescope on a reduced budget ${ }^{2}$. As part of those changes the number of approved science programs was decreased, the funding to the community was decreased and large multi-year programs were encouraged. This has resulted in a decrease in the number of programs per cycle from $\sim 300$ in the cryogenic mission to $\sim 57-125$ in the warm mission. This encouraged astronomers to think in terms of ambitious experiments that require $>500$ hours to accomplish. To date, the largest program that has been accepted is 5286 hours long ${ }^{3}$.

\subsection{Spitzer Publications database}

The Spitzer project maintains a bibliographical database of referred journal articles at http://sohelp2.ipac.caltech.edu/bibsearch. To be included a paper must use Spitzer data as a primary source, or use the Spitzer Legacy and Exploration Science Program's enhanced data products (mosaics, catalogs). We track which observations are used in each paper. In cases where it was not possible to tell exactly what data were used in the paper, we match the paper to all the data that could have been used. Some extra metadata is also tracked to make the database more useful (instrument used, extra-solar planet papers, archival papers, use of enhanced data products) ${ }^{4}$. All numbers in this paper were current as of mid April 2018. At that time, the database contained 7853 papers. A total of 19,768 papers had been inspected to find those, which is a $\sim 40 \%$ signal to noise rate. Note that this paper is only discussing the programs that have an outsized impact on the publication statistics, even though the figures themselves contain all the observations done, to date, with Spitzer.

* escire@,ipac.caltech.edu ; phone: 1 (626) 3953315

Observatory Operations: Strategies, Processes, and Systems VII, edited by Alison B. Peck,

Robert L. Seaman, Chris R. Benn, Proc. of SPIE Vol. 10704, 1070417 · C 2018 SPIE

CCC code: $0277-786 \mathrm{X} / 18 / \$ 18 \cdot$ doi: $10.1117 / 12.2312912$ 


\section{PUBLICATIONS}

\subsection{Number of Papers}

Figure 1 shows the number of papers published per year. It quickly became apparent that Warm Mission observers were not publishing papers the same way they had for the cryogenic mission. In 2014 it was calculated that the average number of hours of observations used in a cryogenic paper was 37.7 while for papers using warm data it was $131.7^{5}$. The warm papers simply use far more data, a reflection of the larger size of the warm mission programs. As a result, we began to also calculate the percentage of exposure time published (Figure 2).

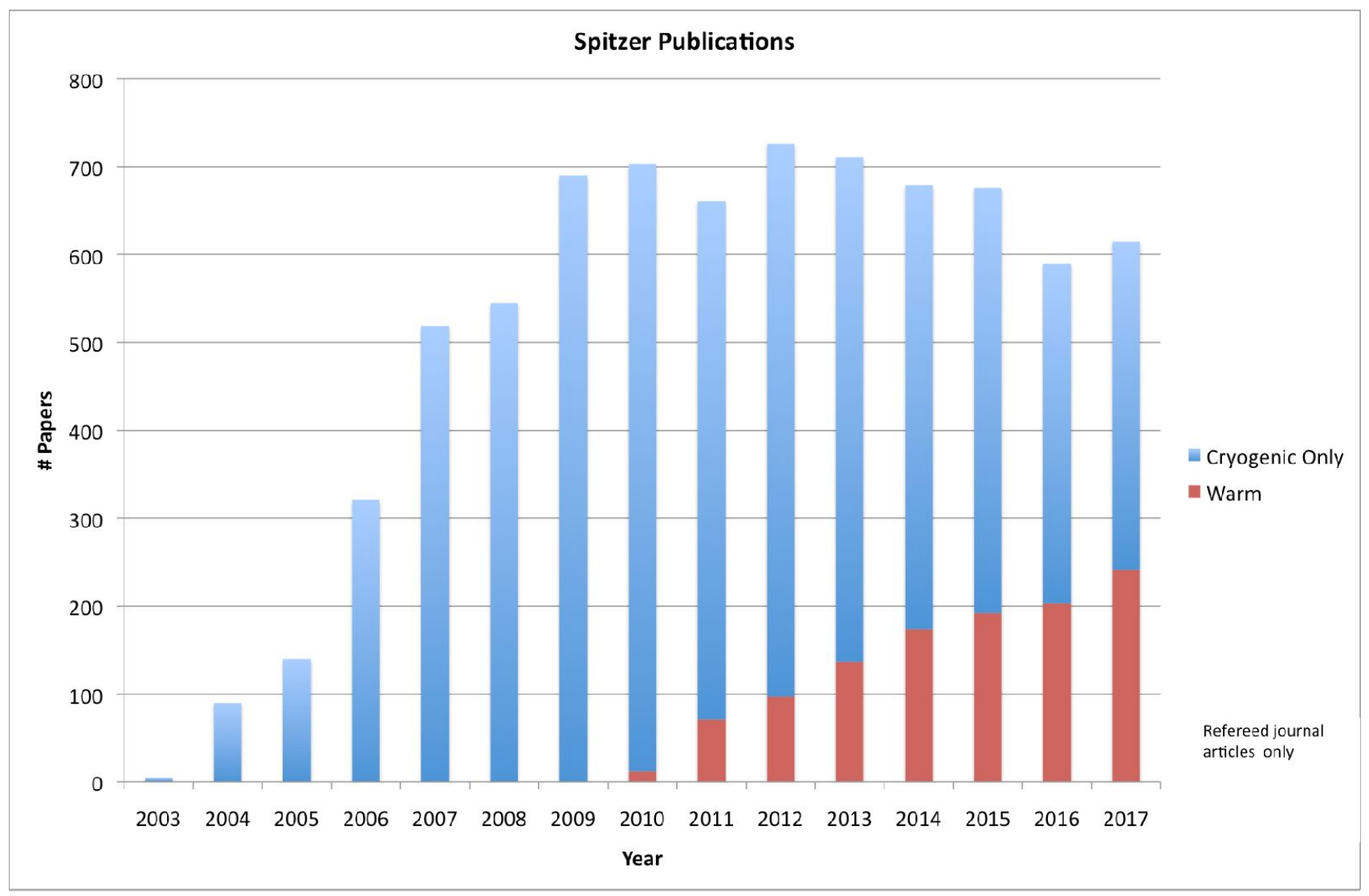

Figure 1. Papers published using Spitzer data as a primary source by year. Warm Mission papers use data taken after the depletion of cryogen in May 2009. A paper that used both warm and cryogenic data is included in the warm bar. 


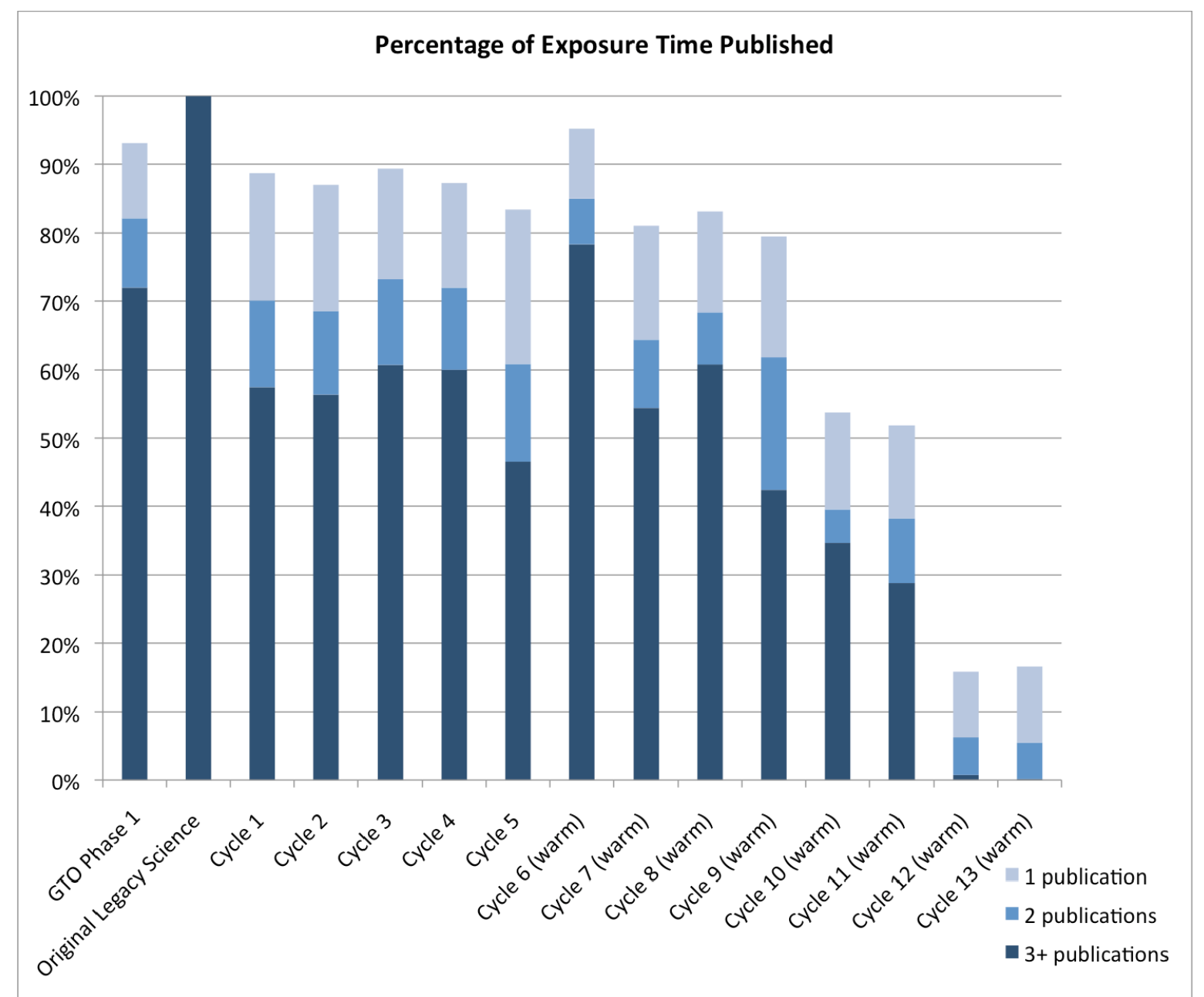

Figure 2. Percentage of exposure time published. Calculated by checking every observation to see how many times it has been published, then normalizing by observation duration. At this time, Cycle 13 is ongoing and so that bar is subject to both negative and positive changes. The categories along the horizontal axis are how the programs are sorted in the operations database: GTO Phase $1+$ Original Legacy Science + Cycle 1 make up the first $\sim 2$ years of the mission. Subsequent cycles have varying durations and sizes (see Figures 5 and 6).

\subsection{Note on enhanced product usage}

If a paper uses all of the data from a program by way of the Legacy/Exploration Science catalogs or mosaics instead of downloading the source data directly and reducing it themselves, then the paper is matched to the program only and not the individual observations (AORs) in the bibliographical database. A program can consist of many AORs. This is done to reduce the workload while matching the papers to their source data and to reduce the size of the publications database, but it has turned out to be a handy way to figure out which papers are using the enhanced data products produced by the Legacy/Exploration Science teams. These papers tend to be of the type where someone is using a deep field catalog to do something statistical, or they are looking at one object in a ultra deep field (since the data in deep fields is stacked to get to the required exposure depth, if you measure the flux from one object you have used all of the data). It can also include an observer using an entire survey to look for a certain types of astronomical object. These papers are NOT included in section 2.3 of this paper, but are added back in section 2.4. If a paper used the enhanced products from a program for a specific object but did not use the entire dataset, then it was matched to the individual AOR and is included in the following sections. The best example of this type of usage is an observer who pulls a value for one star from a catalog that spans the entire Milky Way. 
There are two Director's Discretionary Time (DDT) programs that behave more like Legacy/Exploration Science programs then like DDT ones. They are the First Look Survey (FLS) ${ }^{34,35,36}$ and the Frontier Fields ${ }^{12,13,14,15}$, they have enhanced products and are treated like deep fields in the Spitzer publications database.

\subsection{Time to publish}

\subsubsection{By instrument}

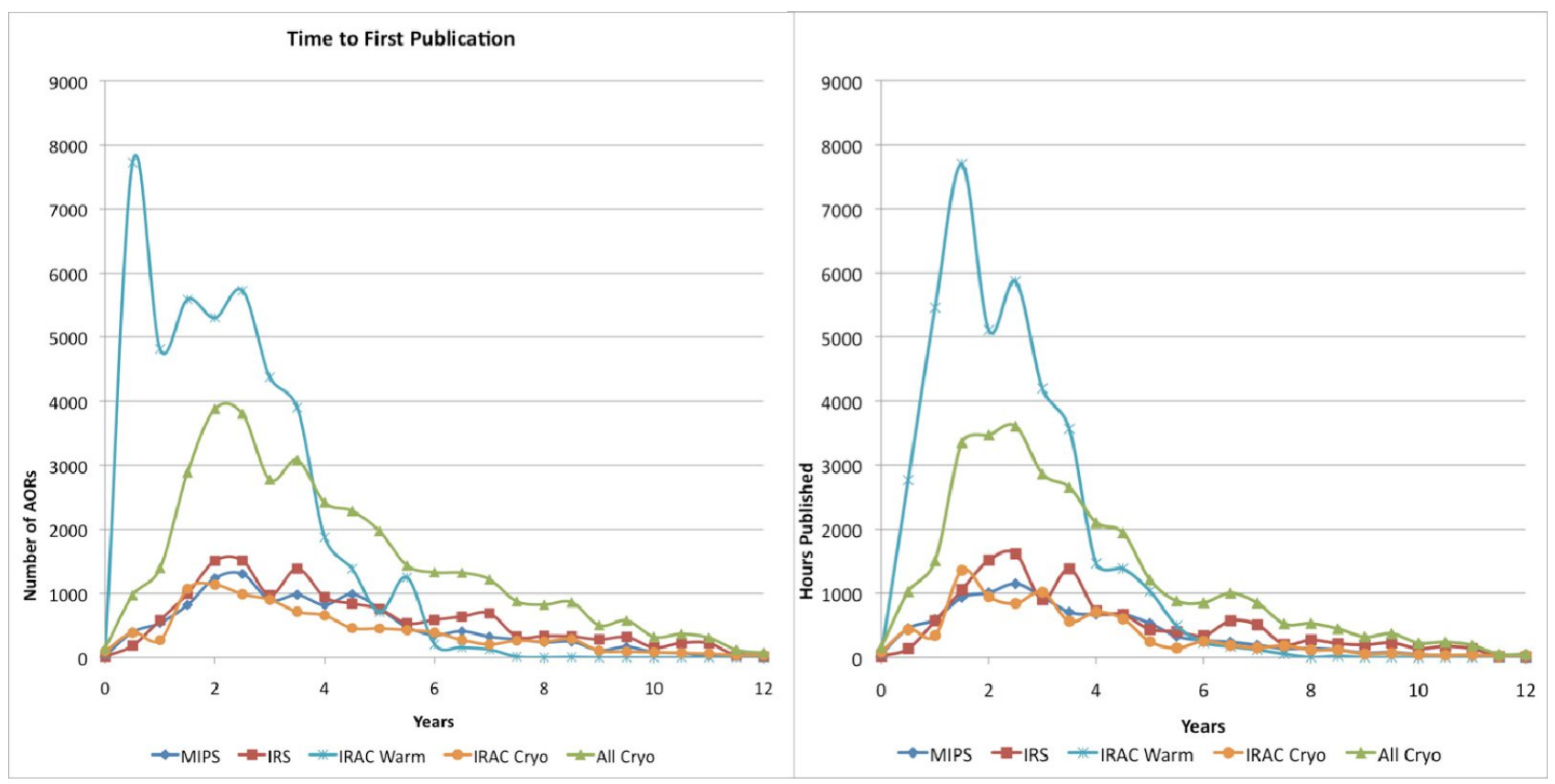

Figure 3. Time elapsed between the data becoming available in the archive and the first publication of the data. The left graph is in AORs and the right one is in hours of observations. Bin size is 6 months. MIPS, IRS and Cryo IRAC together make up the All Cryo line and ceased operations 9 years ago. Warm IRAC has been operating for 9 years.

Figure 3 plots the time it takes for the data to be published for the first time. A paper is considered published when it finishes peer review and is assigned a permanent ADS bibcode. IRAC data is considered easier to use then MIPS and IRS data, which is reflected in the fact that the IRAC Cryo peak is at $\sim 1.75$ years and the IRS and MIPS peaks both come at $\sim 2.25$ years. The large peak in Warm IRAC at 0.5 years in the left hand graph is mostly from one paper. These observations were part of the 2015 Microlensing campaign ${ }^{33}$ conducted for six weeks in the summer of 2015 . The program in question consisted of 5720 individual AORs that averaged 9.5 minutes long each. The observers then used all of the data in the program in one paper published in December of that year ${ }^{6}$. The peak is not present the right hand graph because the observations were so short they only added up to 910 hours of telescope time. The microlensing program would not have been possible during the cryogenic mission.

Figure 4 plots the time to all publications of the data. In the AOR graph you can still see the 2015 Microlensing peak at 0.5 years. Looking at the left hand side of the plots (years 12-14) you can tell the data from the first couple years of the mission are still heavily used today. One interesting feature is that during the cryogenic mission and the very first part of the warm mission, Cryo IRAC, IRS and MIPS were all published at similar rates (years 1-6). After that Cryo IRAC is more republished then MIPS or IRS both when plotting by AORs or by hours (years 7-14). In part this is due to the fact that in the warm mission, only the IRAC instrument is operating and some of the warm programs are expanding upon cryogenic programs by observing more objects, or acquiring new epochs for time domain studies. When those programs are published, they re-use the cryogenic data with the warm data. 


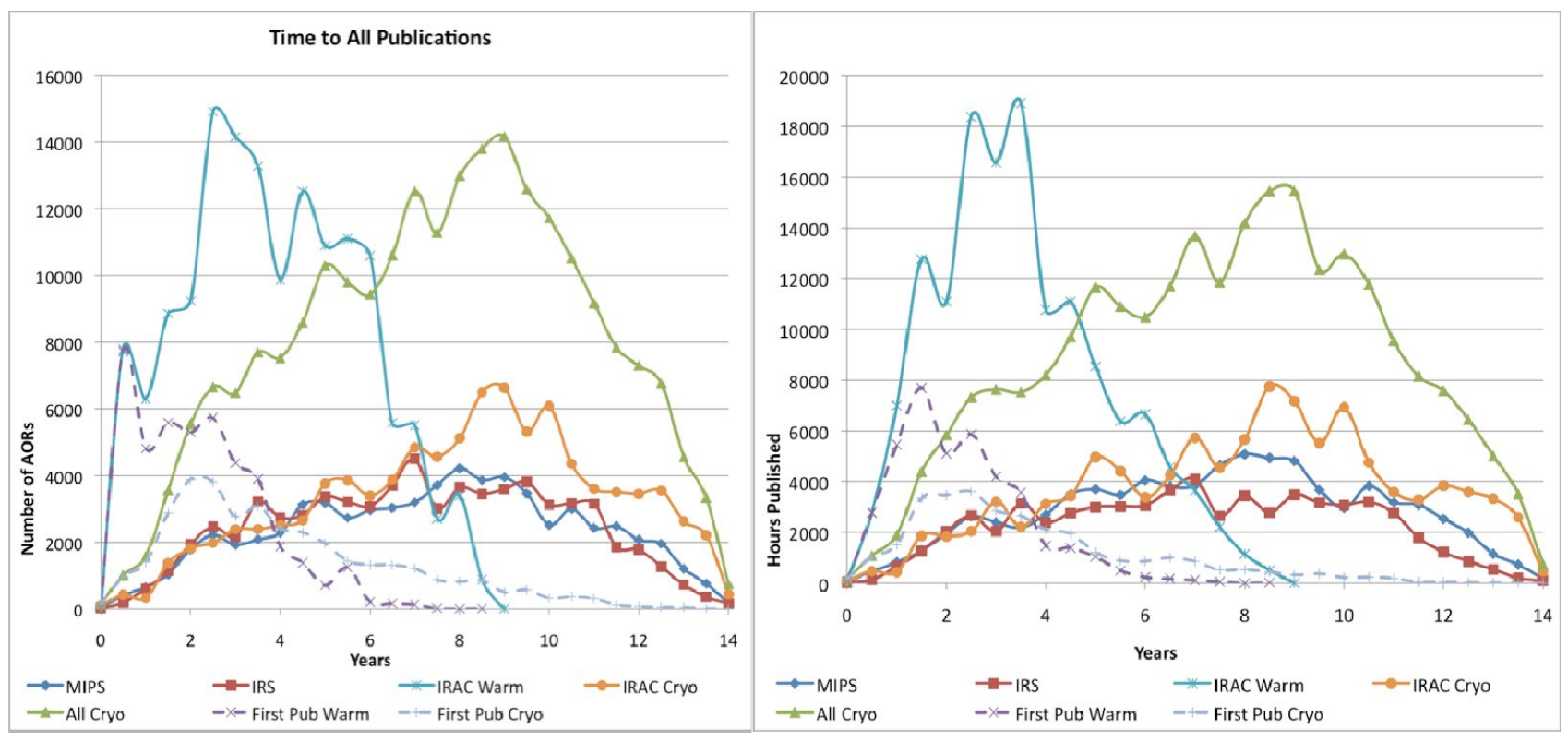

Figure 4. Time elapsed between the data becoming available in the archive and all publications of the data. The left graph is in AORs and the right one is in hours of observations. Bin size is 6 months. MIPS, IRS and Cryo IRAC together make up the All Cryo line and ceased operations 9 years ago. Warm IRAC has been operating for 9 years. The IRAC Warm and All Cryo lines from Figure 3 have been plotting for scale (dotted lines, First Pub Warm and First Pub Cryo, respectively).

Table 1. Programs mentioned in this paper.

\begin{tabular}{|c|c|c|c|c|c|}
\hline \multirow[b]{2}{*}{ Program } & \multicolumn{4}{|c|}{ PROGRAMS MENTIONED IN THIS PAPER } & \multirow[b]{2}{*}{ PI } \\
\hline & Cycle & AORs & Hours & Papers & \\
\hline FLS & GTO Phase 1 & 68 & 112 & 132 & Soifer $^{34,35,36}$ \\
\hline GLIMPSE & Orig Legacy & 393 & 410 & 865 & Churchwell $^{18}$ \\
\hline SINGS & Orig Legacy & 599 & 510 & 626 & Kennicutt $^{9}$ \\
\hline GOODS & Orig Legacy & 212 & 649 & 545 & Dickinson $^{19}$ \\
\hline $\mathrm{C} 2 \mathrm{D}$ & Orig Legacy & 958 & 402 & 507 & Evans $^{22}$ \\
\hline SWIRE & Orig Legacy & 361 & 869 & 453 & Lonsdale $^{23}$ \\
\hline GLIMPSE II & 2 & 314 & 148 & 277 & Churchwell $^{24}$ \\
\hline SAGE & 2 & 198 & 511 & 242 & Meixner $^{25}$ \\
\hline MIPSGAL & 2 and 3 & 539 & 573 & 500 & Carey $^{21}$ \\
\hline SCOSMOS & 2 and 3 & 204 & 614 & 401 & Sanders $^{26}$ \\
\hline GLIMPSE 3D & 3 & 378 & 258 & 218 & Benjamin $^{27}$ \\
\hline FIDEL & 3 & 142 & 396 & 212 & Dickinson $^{28}$ \\
\hline LVLS & 4 & 763 & 277 & 277 & Kennicutt $^{10}$ \\
\hline SAGE-SMC & 4 & 104 & 286 & 95 & Gordon $^{29}$ \\
\hline SDWFS & 4 & 135 & 198 & 91 & Stern $^{30}$ \\
\hline S4G & 6 & 3626 & 637 & 179 & Sheth $^{8}$ \\
\hline SEDS & 6 & 369 & 2104 & 173 & Fazio $^{17}$ \\
\hline CHP & 6 & 3587 & 704 & 80 & Freedman ${ }^{7}$ \\
\hline SERVS & 6 & 820 & 1400 & 75 & Lacy $^{31}$ \\
\hline GLIMPSE 360 & 6 & 2346 & 2141 & 65 & Whitney $^{32}$ \\
\hline SCANDELS & 8 & 281 & 1200 & 91 & Fazio $^{11^{\circ}}$ \\
\hline DEEP GLIMPSE & 8 & 591 & 782 & 48 & Whitney $^{20}$ \\
\hline SPLASH & 9 & 563 & 1272 & 52 & Capak $^{16}$ \\
\hline Frontier Fields 1-4 & 9 & 231 & 871 & 33 & Soifer ${ }^{12,13,14,15}$ \\
\hline 2015 Microlensing & 11 & 5720 & 911 & 12 & Gould $^{33}$ \\
\hline
\end{tabular}




\subsubsection{Sorted by cycle: Graphing by AOR}

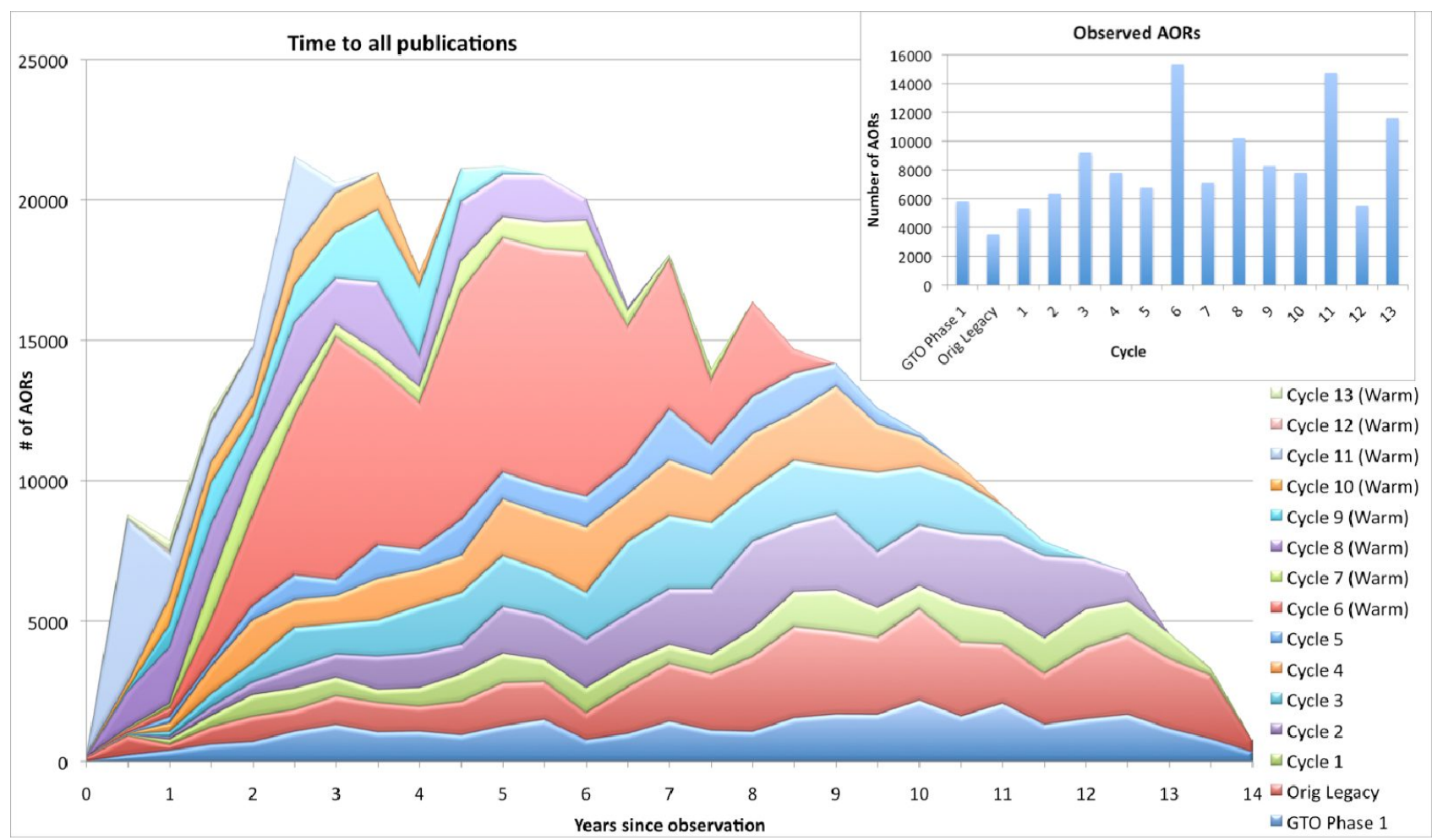

Figure 5. Time elapsed between when the data were released to the Spitzer archive, and all publications of the data, sorted by observing cycle. The vertical axis is number of AORs. The cycles are stacked in ascending order. Insert: The total number of AORs in each cycle. Some cycles were one and half or two year cycles $(6,11,13)$ with a smaller cycle starting one year into them $(7,12)$. At this time Cycle 13 is ongoing.

Over the course of the mission the length of cycles has varied (Figure 5 insert). During the cryogenic mission the cycles were 12 months in duration each with GTO Phase $1+$ Orig Legacy + Cycle 1 running in the first $\sim 2$ years of the mission. In the warm mission Cycles 6 and 11 were both about two years in length with a smaller call (Cycles 7 and 12) issued after a year to take advantage of any science opportunities that cropped up during the year. Cycle 8 was $\sim 16$ months long, Cycle 9 was 12 months, Cycle 10 was 13 months, and Cycle 13 ended up as a two year cycle with two smaller DDT calls that got folded into it in the database. The varying cycle length was determined by the timing of the NASA Senior Reviews where Spitzer applied for Warm Mission extensions.

In addition to varying cycle lengths, there was more program size variance in the warm mission. During the cryogenic mission the operation of three science instruments and $\sim 700-800$ submitted proposals per cycle curtailed most large programs (outside of the Original Legacy Science Call). About 300 cryogenic programs per year were approved, so the available observing time was divided into these many smaller programs. This led to relatively smooth publication trends. You can see in Figure 5 that the cryogenic cycles are roughly even when stacked, with the Original Legacy science cycle holding a slight advantage in numbers of AORs published relative to its small size in AORs (Figure 5 insert).

The scale of science for the warm mission is different. Cycle 6 contained both large programs with long AORs and also two programs with many short AORs that dominate Figure 5: the CHP (Carnegie Hubble Program) ${ }^{7}$ and S4G (Spitzer Survey of Stellar Structure in Galaxies) $)^{8}$. CHP was observing light curves of Cepheid variable stars in order to reduce the uncertainty in the cosmic distance ladder and calculate a more accurate value of the Hubble constant. They published several papers using large chunks of the data set to achieve this goal. After that, some people use small parts of the data for other purposes, but it is not reused in large chucks. This program has 3,587 observed AORs and 18,157 published ones (published/observed ratio $\sim=5 \mathrm{x}$ ).

S4G on the other hand, has a high reuse rate both as individual AORs but also in its entirety as a set. It imaged $\sim 2,300$ nearby $(\mathrm{d}<40 \mathrm{Mpc})$ galaxies and is used by people who are interested in studying galaxy structure, as well as people 
bridging the gap between studying nearby galaxies and those studying deep fields. It can be seen as the continuation of two cryogenic mission programs: the Spitzer Infrared Nearby Galaxies Survey (SINGS) ${ }^{9}$ (Original Legacy) and the Local Volume Legacy Survey (LVLS) ${ }^{10}$ (Cycle 4). Since it is used as a statistical set and it has thousands of AORs, each paper that is published using S4G data changes the shape of Cycle 6 in Figure 5 dramatically. This program has 3626 observed AORs and 41,417 published ones (ratio: 11.4x).

Once again in Figure 5 the 2015 Microlensing peak shows up in Cycle 11 at 0.5 years.

One side effect of having less programs per cycle is that publication rates are highly dependant on a fewer number of observers. This makes them less predicable because if the team from one large program is delayed for any reason, a large portion of data is less useful then it could be if catalogs and mosaics had been generated in a timely manner. Another side effect of large multi-year programs is a significant delay in the data taken early in the cycle being used. If the science goals depend on having the entire data set, the observer might have to wait for two years to get the rest of their data before producing their enhanced products.

\subsubsection{Sorted by cycle: Graphing by Hours}

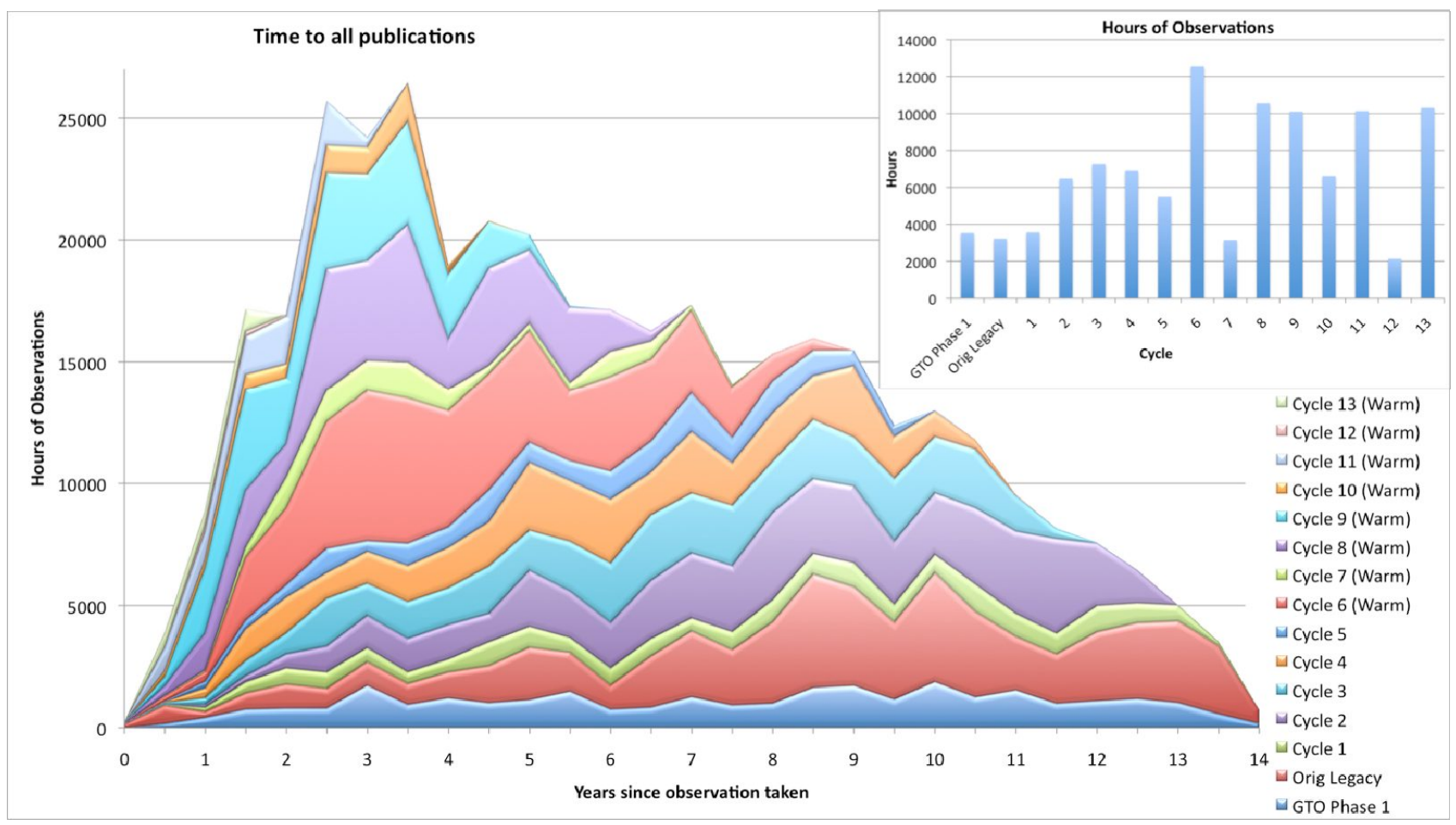

Figure 6. Time elapsed between when the data were released to the Spitzer archive, and all publications of the data, sorted by observing cycle. The vertical axis is hours of observations. The cycles are stacked in ascending order. Insert: total hours of observations in each cycle. At this time Cycle 13 is ongoing.

If you plot the time to publish normalized by the duration of each observation (Figure 6) then programs with large numbers of hours but fewer AORs start to dominate. These programs tend to be deep fields programs with allocations in the thousands of hours, but only a few hundred AORs. The 2015 Microlensing peak disappears, Cycle 6 looks less outsized, and the impact of Cycles 8 and 9 grows. Cycle 8 is home to the S-CANDELS deep fields ${ }^{11}$, and Cycle 9 contained 4 of the 6 Frontier Fields ${ }^{12,13,14,15}$ and the first part of SPLASH (Spitzer Large Area Survey with HyperSuprime-Cam) ${ }^{16}$ (both of these programs continued into Cycle 10).

When normalized by hours, the influence of CHP and S4G on the shape of the graph decreases and the shape of Cycle 6 becomes driven by SEDS (Spitzer Extended Deep Survey) ${ }^{17}$. This program imaged five deep fields in 2105 hours and currently has 21,482 published hours of data (ratio: 10.2x).

Also, the relative impact of the Original Legacy Science programs becomes apparent. GTO Phase 1, Original Legacy and Cycle 1 were all about the same size in number of hours and were all observed at the same time, yet the data from 
the Original Legacy programs are republished at a much higher rate. The three programs that dominate the continued publication of the Original Legacy data are the Galactic Legacy Infrared Mid-Plane Survey Extraordinaire (GLIMPSE) $^{18}$, the Great Observatories Origins Deep Survey (GOODS) $)^{19}$ and SINGS ${ }^{9}$.

GLIMPSE is a mapping program with IRAC to image the Milky Way that spans many cycles: Original Legacy Science, Cycles 2, 3, 6 and 8. The Original Legacy science part of GLIMPSE mapped the middle third of the Milky Way excluding the galactic center and is the highest used part of that dataset. It may be that this is true because this part of the Milky Way has many more astronomical sources then the outer reaches or because there is more complementary data from other observatories in that region. The galactic center was mapped by GLIMPSE $\mathrm{II}^{24}$ in cycle 2. Its companion program, MIPSGAL ${ }^{21}$, mapped part of the Milky Way with MIPS during Cycles 2 and 3. These two programs are heavily used to this day both as individual observations by scientists looking for more data on their specific object, and also as an entire dataset by scientists doing larger scale science. The Original Legacy part of GLIMPSE has 409 observed hours and has 19,750 published hours (ratio: 48x), MIPSGAL cycle 2 has 417 observed hours and 15,146 published hours (ratio: $36.4 \mathrm{x}$ ).

SINGS imaged 75 nearby galaxies $(\mathrm{d}<30 \mathrm{Mpc})$ with IRAC and MIPS, and took IRS spectra "of their centers and a representative set of extranuclear IR-emitting regions in the galaxies"". This dataset is sometimes used in its entirety, but it tends to be used one galaxy at a time, and is referenced when scientists look at any object in those galaxies. The sample size was expanded in Cycle 4 with LVLS, and Cycle 6 with S4G. Both of these programs are also heavily used which have helped to expand the usage of SINGS. SINGS has 511 observed hours and 11,264 published ones (ratio: 22x).

GOODS imaged the Chandra Deep Field South and the Hubble Deep Field North with IRAC and MIPS. Due to the continued high interest in those fields and ongoing observations with other telescopes, catalogs of these fields are produced regularly. GOODS has 649 observed hours and 18,324 published ones (ratio: 28.2x). We track catalog usage of the deep fields separately so the above graphs only include usage where people downloaded the source data and reduced it themselves.

\subsection{Enhanced Products usage}

In this section we add back in the papers that use the entire Legacy/Exploration Science enhanced products (see section 2.2). It becomes very apparent in Figure 7 that having these products is a very good thing and they are one of the primary ways that data, especially deep field data, gets used. 


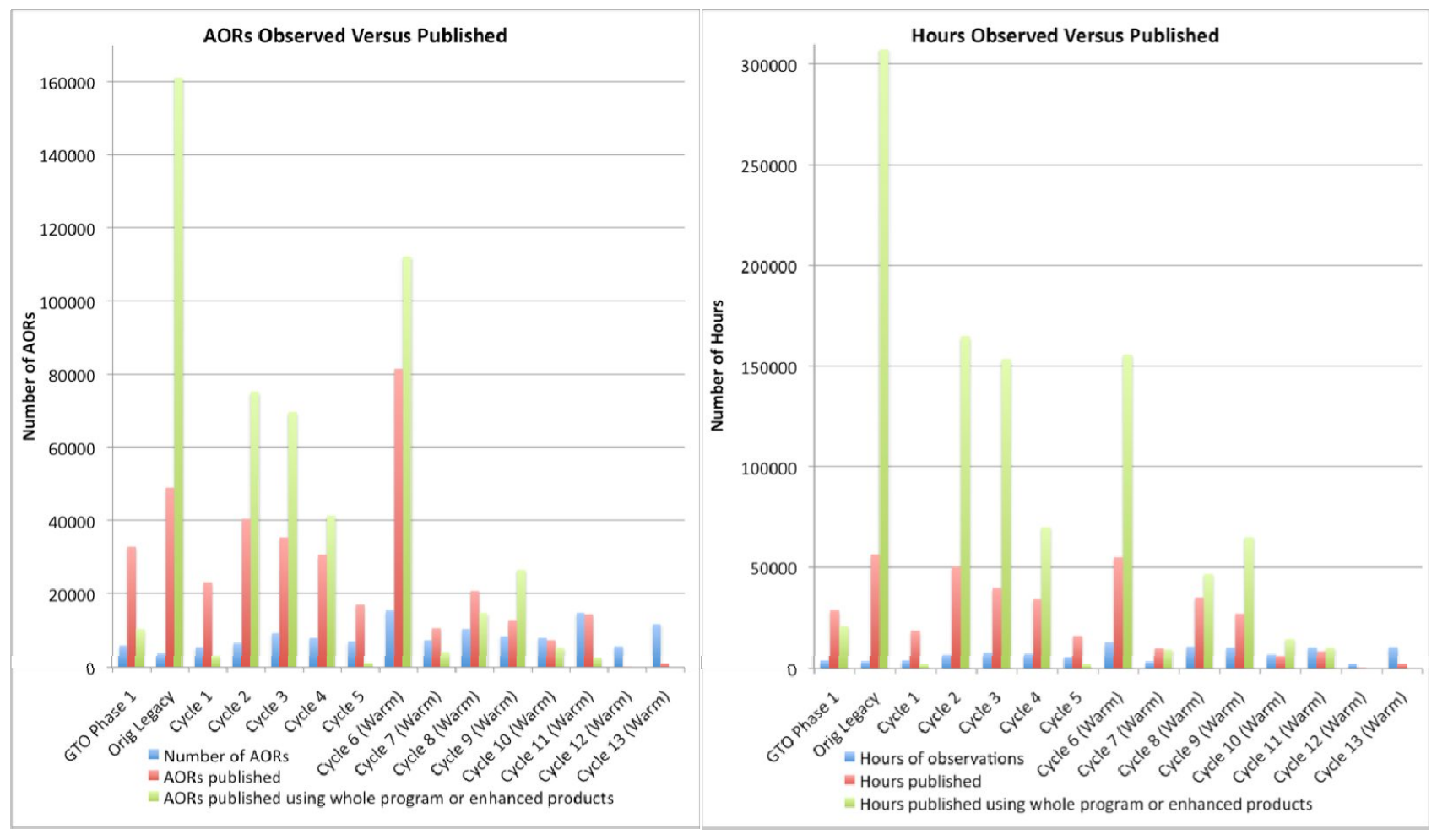

Figure 7. Total observed and published observations by cycle. The left panel is in units of AORs, the right one is normalized by hours. The blue bar on both is the observations taken with Spitzer, the red bar is the sum of the observations published, and the green bar is the Legacy/Exploration Science enhanced products usage (See Section 2.2). The enhanced products usage is computed by multiplying the total number of papers that use the enhanced products by the number of AORs or total hours in used to create those products. Table 2 breaks down the enhanced products bars by program.

Table 2. Enhanced products usage in Fig. 7. The middle two columns are the percentage of the enhanced products bar that the program contributes when calculated in AORs or by hours. Only programs that contribute significantly to Fig. 7 are included in this table.

\begin{tabular}{|lrlr|}
\hline \multicolumn{5}{|c}{ ENHANCED PRODUCTS USAGE IN FIGURE 7} & Cycle \\
Program & AORs & Hours & GTO Phase 1 \\
FLS - Extragalactic ${ }^{34}$ & $89 \%$ & $94 \%$ & Original Legacy \\
GOODS & $37 \%$ & $57 \%$ & Original Legacy \\
SWIRE & $25 \%$ & $30 \%$ & Original Legacy \\
GLIMPSE & $12 \%$ & $6 \%$ & Original Legacy \\
C2D & $13 \%$ & $3 \%$ & 2 \\
SCOSMOS & $32 \%$ & $44 \%$ & 2 \\
SAGE & $33 \%$ & $37 \%$ & 2 \\
MIPSGAL & $12 \%$ & $6 \%$ & 2 \\
GLIMPSE II & $17 \%$ & $4 \%$ & 3 \\
SCOSMOS & $42 \%$ & $53 \%$ & 3 \\
FIDEL & $30 \%$ & $36 \%$ & 3 \\
GLIMPSE 3D & $17 \%$ & $5 \%$ & 4 \\
SAGE-SMC & $21 \%$ & $46 \%$ & 4 \\
SDWFS & $28 \%$ & $32 \%$ & 4 \\
LVLS & $42 \%$ & $12 \%$ & 6 \\
SEDS & $16 \%$ & $66 \%$ & 6 \\
SERVS & $12 \%$ & $15 \%$ & 6 \\
GLIMPSE360 & $15 \%$ & $10 \%$ & 6 \\
S4G & $54 \%$ & $7 \%$ & 8 \\
SCANDLES & $63 \%$ & $85 \%$ & 8 \\
DEEPGLIMPSE & $16 \%$ & $6 \%$ & 9 \\
SPLASH & $91 \%$ & $84 \%$ & \\
\hline
\end{tabular}




\subsection{Other graphs}

This section contains other graphs breaking down the publications by instrument (Figure 8), data use from the Legacy and Exploration Science programs (Figure 9) and archival publications (Figure 10).

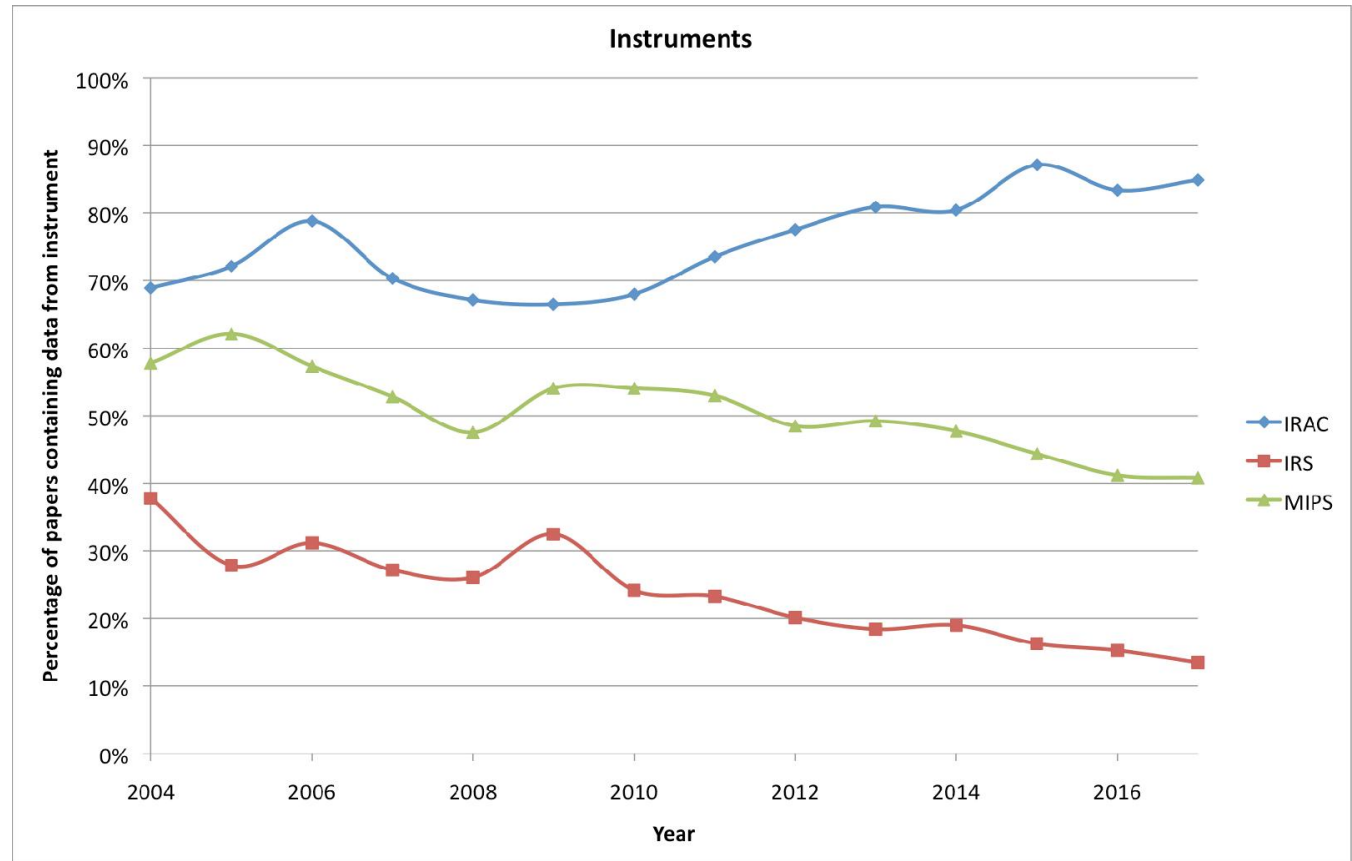

Figure 8. Percentage of Spitzer papers using data from the IRS, MIPS and IRAC instruments. Since 2009, only the IRAC instrument has been operational.

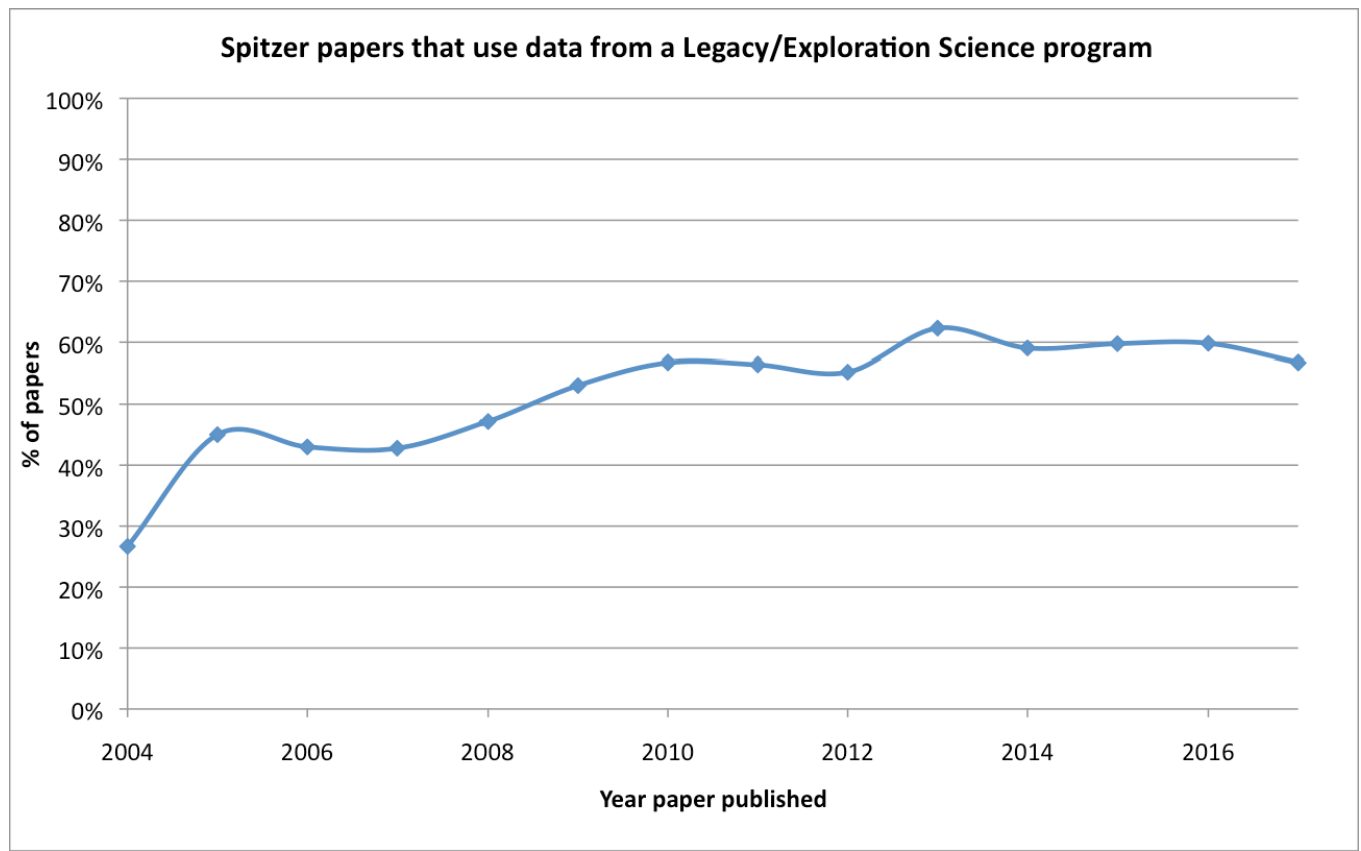

Figure 9. Spitzer papers that use data (either raw data or enhanced products (catalogs/mosaics)) from an Exploration Science or Legacy program.

Proc. of SPIE Vol. 10704 1070417-10 


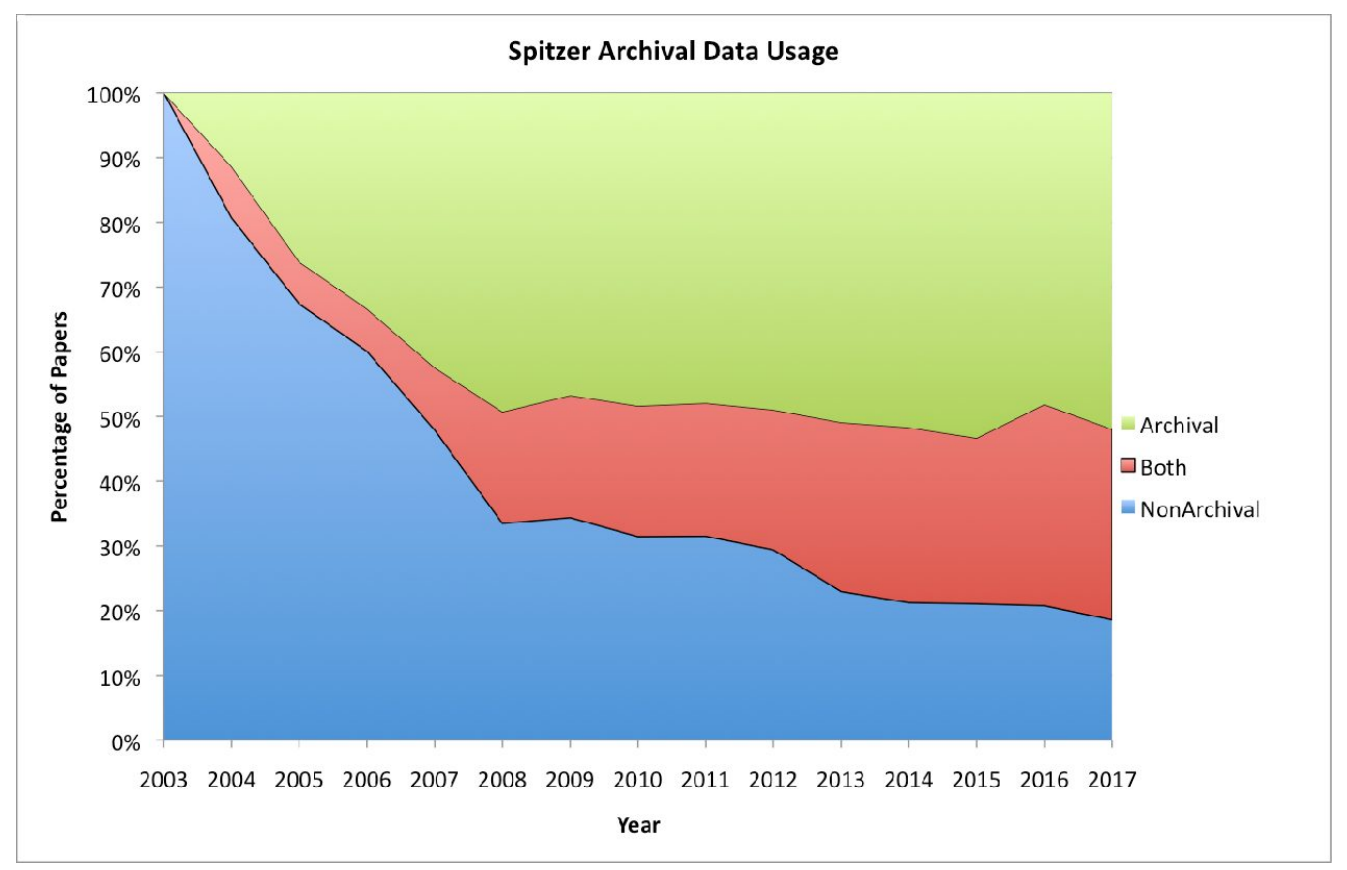

Figure10. Archival Spitzer papers. To calculate this the last names of the paper's authors were compared to the last names of all people on an observing proposal. A NonArchival paper has at least one match for all programs used in the paper. An Archival paper is one where no authors match anybody on the proposal. Papers classified as both use data from multiple programs and have at least one program that is archival, and one program that is non-archival.

\section{CONCLUSION}

As the Spitzer mission changed to cope with a reduced budget, the programs selected changed which in turn has changed how the data are used and published. It is less straightforward to graph publications in the warm mission then it was during the cryogenic one, and knowing about the programs themselves and how observers use them is essential for decoding what is happening to the papers. While there are less warm mission papers then cryogenic mission papers the warm data is still heavily used. The warm papers use about 3.5 times more data per paper then cryogenic papers. In addition, due to larger program sizes, the warm mission data is not published at a smooth rate, it is published in larger chucks. Because some warm mission programs require the entire dataset before publication, there can be a publication delay for data taken early in a cycle. When plotting the time to the first publication of the data the peak is at $\sim 2$ years and does differ by instrument with IRS and MIPS being somewhat slower then Cryo and Warm IRAC. The cryogenic data, especially the Original Legacy Science programs, are still heavily used even though the cryogenic mission ended 9 years ago and the Original Legacy Science data was taken 14 years ago. This is driven in part by warm mission programs that expand sample sizes or obtain more epochs of objects from cryogenic mission programs. Data from the Legacy and Exploration Science programs is used in $\sim 55 \%$ of Spitzer papers, and generating enhanced data products and having a good archive can substantially increase the publication rates. Some fields on the sky produce more papers then others, and in general the time allocation committees over the lifetime of the Spitzer mission did an excellent job in selecting programs that have lasting legacy value.

\section{ACKNOWLEDGEMENTS}

This work is based on observations made with the Spitzer Space Telescope, which is operated by the Jet Propulsion Laboratory, California Institute of Technology under a contract with NASA. 


\section{REFERENCES}

[1] Werner, M. et al., "The Spitzer Space Telescope Mission," ApJS 154, 1-9 (2004).

[2] Storrie-Lombardi, L. J., Dodd, S. R., "Downsizing a great observatory: reinventing Spitzer in the warm mission," Proc. SPIE 7737, 77370L (2010).

[3] Capak, P. et al., "The Euclid/WFIRST Spitzer Legacy Survey," Spitzer Proposal 13058 (2016).

[4] Scire, E., Chan, B. H. P., Silbermann, N., Shields, A., "The Spitzer Bibliography Database: bibliographic statistics," Proc. SPIE 7737, 77371V (2010).

[5] Scire, E., "Warm Spitzer: Effects of Major Operational Changes on Publication Rates," ASPC 485, 481 (2014).

[6] Calchi Novati, S. et al., "Spitzer IRAC Photometry for Time Series in Crowded Fields," ApJ 814, 92 (2015).

[7] Freedman, W., Madore, B., Mager, V., Persson, E., Rigby, J., Sturch, L., “The Hubble Constant,” Spitzer Proposal 60010 (2008).

[8] Sheth, K. et al., "The Spitzer Survey of Stellar Structure in Galaxies (S4G)," Spitzer Proposal 60007 (2008).

[9] Kennicutt, R. et al., "SINGS: The Spitzer Infrared Nearby Galaxies Survey - Physics of the Star-Forming ISM and Galaxy Evolution," Spitzer Proposal 159 (2004).

[10] Kennicutt, R. et al., "The Local Volume Legacy Survey,” Spitzer Proposal 40204 (2007).

[11] Fazio, G. et al., "Spitzer Very Deep Survey of the HST/CANDELS Fields," Spitzer Proposal 80057 (2011).

[12] Soifer, T., Capak, P., "Frontier Field A2744," Spitzer Proposal 90257 (2013).

[13] Soifer, T., Capak, P., "Frontier Field MACS J0416," Spitzer Proposal 90258 (2013).

[14] Soifer, T., Capak, P., "Frontier Field MACS J0717," Spitzer Proposal 90259 (2013).

[15] Soifer, T., Capak, P., "Frontier Field MACS J1149," Spitzer Proposal 90260 (2013).

[16] Capak, P. et al., "SPLASH: Spitzer Large Area Survey with Hyper-Suprime-Cam," Spitzer Proposal 90042 (2012).

[17]Fazio, G. et al., "SEDS: The Spitzer Extended Deep Survey," Spitzer Proposal 60022 (2008).

[18] Churchwell, Ed et al., "The SIRTF Galactic Plane Survey," Spitzer Proposal 146 (2004).

[19] Dickinson, M. et al., "Great Observatories Origins Deep Survey (GOODS)," Spitzer Proposal 169 (2004).

[20] Whitney, B. et al., "Deep GLIMPSE: Exploring the Far Side of the Galaxy," Spitzer Proposal 80074 (2011).

[21] Carey, S. et al., "MIPSGAL: A 24 and 70 Micron Survey of the Inner Galactic Disk with MIPS," Spitzer Proposal 20597 (2005).

[22] Evans, N. et al., "From Molecular Cores to Planets," Spitzer Proposal 139 (2004).

[23] Lonsdale, C. et al., “ The SIRTF Wide-area InfraRed Extragalactic Survey,” Spitzer Proposal 142 (2004).

[24] Churchwell, Ed et al., "GLIMPSE II: Imaging the Central +/-10 Degrees of the Galactic Plane with IRAC," Spitzer Proposal 20201 (2005).

[25] Meixner, M. et al., "Spitzer Survey of the Large Magellanic Cloud: Surveying the Agents of a Galaxy's Evolution (SAGE)," Spitzer Proposal 20203 (2005).

[26] Sanders, D. et al., "S-COSMOS: The Spitzer Deep Survey of the HST COSMOS 2-Degree ACS Field," Spitzer Proposal 20070 (2005).

[27] Benjamin, R. et al., "GLIMPSE-3D: The Vertical Stellar and Interstellar Structure of the Inner Galaxy," Spitzer Proposal 30570 (2006).

[28] Dickinson, M. et al., "A Deep-Wide Far-Infrared Survey of Cosmological Star Formation and AGN Activity," Spitzer Proposal 30948 (2006).

[29] Gordon, K. et al., "SAGE-SMC: Surveying the Agents of Galaxy Evolution in the Tidally-Disrupted, LowMetallicity Small Magellanic Cloud," Spitzer Proposal 40245 (2007).

[30] Stern, D. et al., "SDWFS: The Spitzer Deep, Wide-Field Survey," Spitzer Proposal 40839 (2007).

[31] Lacy, M. et al., "SERVS: The Spitzer Extragalactic Representative Volume Survey," Spitzer Proposal 60024 (2008).

[32] Whitney, B., "GLIMPSE360: Completing the Spitzer Galactic Plane Survey," Spitzer Proposal 60020 (2008).

[33] Gould, A., Carey, S. Yee, J., "Galactic Distribution of Planets from Spitzer Microlens Parallaxes," Spitzer Proposal 11006 (2014).

[34] Soifer, T., Storrie-Lombardi, L., "First Look Survey - Extragalactic Component," Spitzer Proposal 26 (2003).

[35] Soifer, T., Storrie-Lombardi, L., "First Look Survey - Ecliptic Plane Component," Spitzer Proposal 98 (2003).

[36] Soifer, T., Storrie-Lombardi, L., "First Look Survey - Galactic Component," Spitzer Proposal 104 (2003). 